\title{
Rotigotine in Parkinson's Disease Patients: What is the Efficient and Tolerable Dose According with the Real Clinical Practice? An Open, Non-Controlled Multicenter Spanish-Study
}

\author{
Salazar G*, Fragoso M and Codas J \\ Department of Neurology, Hospital Terrassa CST, Terrassa, Barcelona, Spain
}

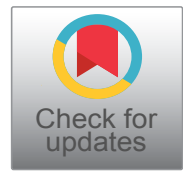

*Corresponding author: Gabriel Salazar, Department of Neurology, Hospital Terrassa CST, Terrassa, Barcelona, Spain, Tel: 34-607471876,E-mail: gsalazar@cst.cat

\begin{abstract}
Background: The efficacy and the tolerance of Rotigotine in PD patients has been shown in some studies published in the past. Nevertheless, there is few information in regards to the dose of Rotigotine used in the real clinical practice.
\end{abstract}

Methods: We designed a multicenter, non-controlled, open follow up to study the efficient and tolerable doses of Roti-gotine used in the real clinical practice in mild impaired PD patients.

Results: Patients included in this study showed a significant improvement in regards to the UPDRS motor scale, as well as in the PDQ-8 functional scale and the patient global impression scale at the end of the study. Whereas, the score in global clinical impression as well as in the NPI scale showed no significant statistical improvement at the end of the end of the study.

Conclusions: PD patients of this study showed a motor and functional improvement after 3 months of Rotigotine transdermic patches therapy with a mean dose of $7.6 \mathrm{mg} /$ daily and they experienced few and non-relevant adverse effects. the neuropsychiatric symptoms and impulse control disorders were rarely seen after Rotigotine therapy at $8 \mathrm{mgs} /$ daily.

\section{Keywords}

Parkinson, Rotigotine, Dopaminergic agonist

\section{Introduction}

Rotigotine is dopaminergic agonist (DA) with a high affinity for D3-dopaminergic receptors delivered through transdermal patch which has shown efficacy and tolerance in Parkinson's disease patients (PD) according with some articles published in the past [1]. Whereas, most of the studies published on Rotigotine have shown effi- cacy to ameliorate the cardinal symptoms of PD at daily dose of $8 \mathrm{Mgs}$ [2] others have shown similar results with a variable range of dally dose of Rotigotine from 6 to $16 \mathrm{mg}$ [3-5]. There are few studies of real clinical practice with Rotigotine in patients with PD which describe doses of this DA after the well-known pivotal studies. We designed a multicentric- prospective-open label-no control study to evaluate Rotigotine dosing in early PD patients of 8 Spanish hospitals in a real clinical practice study.

\section{Material and Methods}

PD patients with mild clinical symptoms and functional impairment who required therapeutic intervention according with the clinical practice and coming from the movement's disorders units of 8 Hospital of Catalonia, Spain were included into the study. All the patients fulfilled the brain bank Queen square criteria for parkinson's disease, with or without I-dopa treatment, and should require an adjustment of therapy in the screening visit, following the clinical criteria of the neurologist. Patients started Rotigotine in patch with 2 mgs with a progressive weekly increment of $2 \mathrm{Mgs}$ up to $8 \mathrm{Mgs} /$ day. The I-dopa dose was stable, no changes were allowed, during the 12 weeks of follow up.

Patients were visited every three weeks during this 12 -weeks of follow up. Screening visit (SV), basal visit (VO), visit 1 (V1) and visit 2 (V2).

All patients underwent to some common PD motor and functional scales (1) UPDRS III, (2) Hoehn and Yahr 


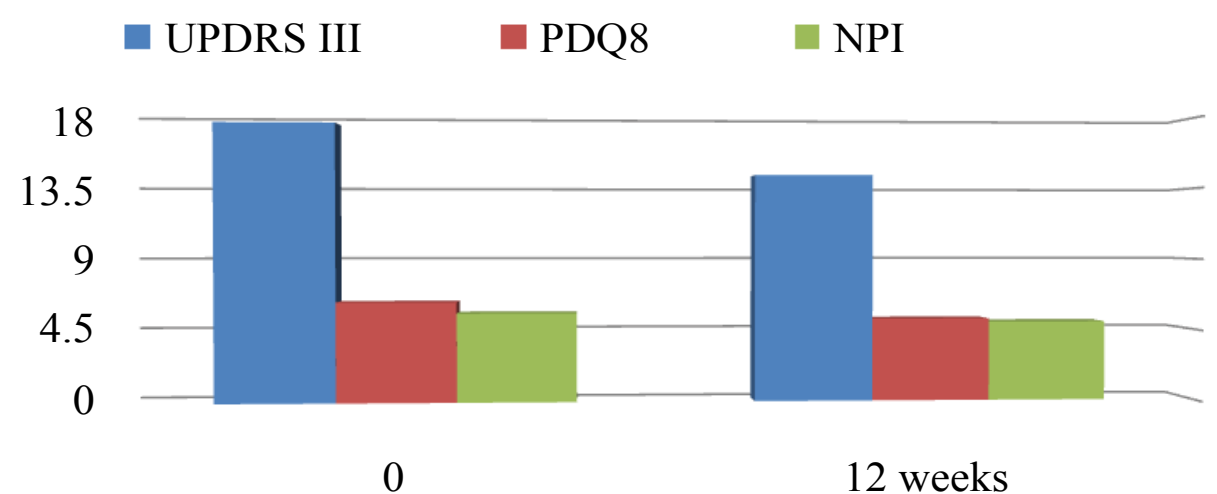

Figure 1: UPDRS III, PDQ8 and NPI scales, basal and three months evaluations after Rotigotine in patch.

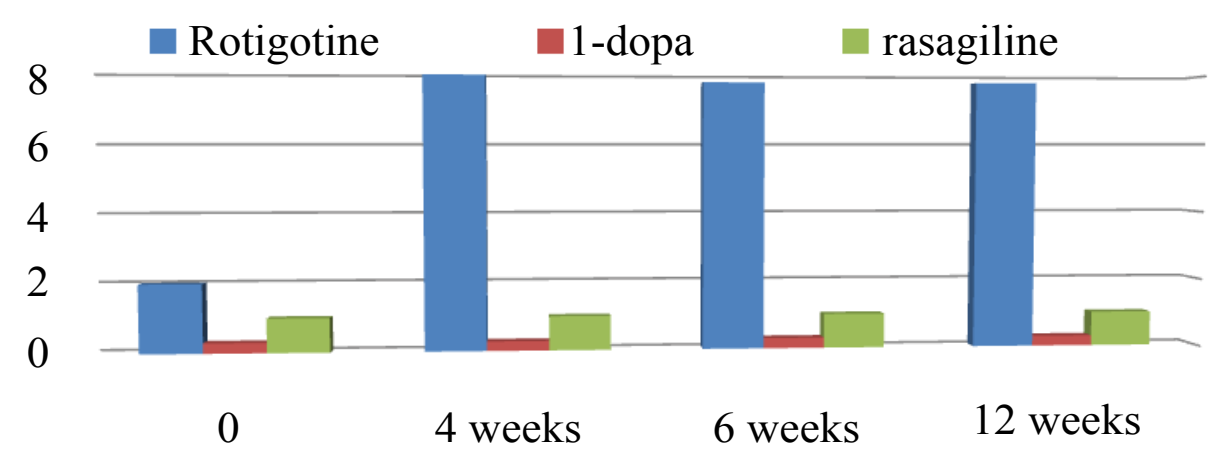

Figure 2: Dosing of Rotigotine, L-Dopa and Rasagiline in a twelve weeks (3 months) follow up.

Scale, (3) Neuropsychiatric inventory (NPI), (4) PDQ8 scale, (5) Global impression scale for doctors (GIC) and (6) Global impression scale for patients (GIP) at the basal and final evaluations.

Psychiatrics and physical side effects were actively asked to all the patients.

The data were analyzed comparatively among both visits, basal and final visit. the value of significance was $p<0.05$, paired-t student and Wilcoxon signed rank tests were used and McNemar's test for proportions.

\section{Results}

42 PD patients coming from 8 hospitals of Catalonia, Spain were included into the study and all of them were followed up until the end of the study.

Patients showed in the UPDRS part III sub-scale, a mean reduction in the score from 17.8 to 14.5 points, $(-18.6 \%)(p<0.05)$ at the of the study. In the NPI scale, they showed a mild reduction in the score from 5.6 to 5 points at the end of the study $(-10.7 \%)(p>0.05)$. In the PDQ 8-Scale, they showed a significant reduction in the score from 6.3 to 5.2 (17.5\%) ( $p>0.05$ ) (Figure 1). In regards to the global clinical impression $(\mathrm{GCl})$, neurologists considered that $52.1 \%$ of patients were mild sick and $42 \%$ moderately sick at the basal evaluation, but at the end of the follow up, neurologists considered that PD patients were $68 \%$ mild sick and $32 \%$ moderately sick at the twelve weeks evaluation, at the end of the study, therefore, the difference was not statistically significant $(p>0.05)$, whereas, the Global impression scale for the patients (GIP): $41.3 \%$ of them reported mild sick and $58.7 \%$ of them moderately sick at the basal self report evaluation but at the end of the study, $69 \%$ of them reported mild sick and $31 \%$ moderately sick which represents a significant reduction of the score in the GIP scale at the 12-weeks evaluation ( $p>0.05)$.

The mean dose of Rotigotine was of $7.6 \mathrm{mg} /$ day at the end of the study, the most frequently used dose was $8 \mathrm{mg} /$ day ( $87 \%$ of the cases). 2 patients (4.7\%) presented mild adverse effects which consisted in mild topical rash in the area of the patch (Figure 2).

\section{Discussion}

The efficacy and the tolerance of Rotigotine in PD patients has been shown in some studies published in the past [6]. Nevertheless, there is few information in the medical literature in regards to the dose of Rotigotine used in the real clinical practice to determine what is the border between the good clinical effects and the possible adverse effects in our daily clinical practice [7]. PD patients included in this study showed a significant improvement in regards to the UPDRS motor scale, sub item III as well as in the PDQ-8 functional scale at the end of the study. This improvement was expressed for the patients in the self-reported global impression scale at the final evaluation, however, the global clinical impression reported for the evaluators showed a non significant improvement at the end of the study compared to the basal visit, we can attribute this data to the mild 
functional impairment of the PD patients population included in this study, therefore, it is difficult to determine an important motor improvement, whereas, PD patients of this study showed a clear functional improvement in the PDQ-8 functional scale after three month of Rotigotine therapy, probably the improvement in non-motor symptoms such as depression, insomnia and fatigue can explain this data.

When we compared PD patients with and without I-dopa therapy, both group showed no statistical significant differences between them in regards to all the primary assessment measures.

In regards to neuropsychiatrics symptoms, PD patients showed no changes after Rotigotine treatment at the end of the study, we can observe in the NPI scale a non-significant changes reported at the end of the follow up, therefore, we want to remark this data as relevant because of the higher risk of neuropsychiatric symptoms reported with all the dopaminergic agonists.

\section{Conclusions}

PD patients of this study showed a motor and functional improvement after 3 months of Rotigotine transdermic patches therapy with a mean dose of $7.6 \mathrm{Mgs} /$ daily. Patients experienced few and non-relevant adverse effects. the neuropsychiatric symptoms and impulse control disorders were rarely seen after Rotigotine therapy at $7.6 \mathrm{Mgs} /$ daily. We are aware that this open and non-controlled study has important scientific limitations but it represents a good sample of our Eu- ropean PD population who are in treatment with Rotigotine in patch and help us to observe the efficacious and tolerable dose of Rotigotine used in the real clinical practice out of the original pivotal studies.

\section{References}

1. Giladi N, Boroojerdi B, Surmann E (2013) The safety and tolerability of rotigotine transdermal system over to 6-year period in patients with early-stage parkinson's disease. $J$ Neural Transm (vienna) 120: 1321-1329.

2. Nicholas AP, Orgohain R, Chaná $P$, Surmann E, Thompson EL, et al. (2014) Randomized study of rotigotine dose response on 'off' time in advanced parkinson's disease. J Parkinsons Dis 4: 361-373.

3. Rektor I, Babic T, Boothmann B, Polivka J, Boroojerdi B, et al. (2009) High doses of rotigotine transdermal patch: results of an open-label, dose-escalation trial in patients with advanced-stage, idiopathic parkinson disease. Clin Neuropharmacol 32: 193-198.

4. Watts R, Jankovic J, waters C, Rajput T, Boroojerdi B, et al. (2007) Randomized, blind, controlled trial of transdermal rotigotine in early parkinson disease. Neurology 68: 272-276.

5. Parkinson Study Group (2003) A controlled trial of rotigotine monotherapy in early parkinson's disease. Arch Neurol 60: 1721-1728.

6. Güldenpfennig W, Poole K, Sommerville K, Boroojerdi B (2005) Safety, tolerability, and efficacy of continuous transdermal dopaminergic stimulation with rotigotine patch in early- stage idiopathic parkinson disease. Clin Neuropharmacol 28: 106-110.

7. Alonso Cánovas A, Luquin Pulido R, García Ruiz P, Burguera JA, Campos Arillo V, et al. (2014) The Dopaminergic Agonists in Parkinson's disease. Neurologia 29: 230-241. 